\title{
Intestinal flora imbalance induced by antibiotic use in rats
}

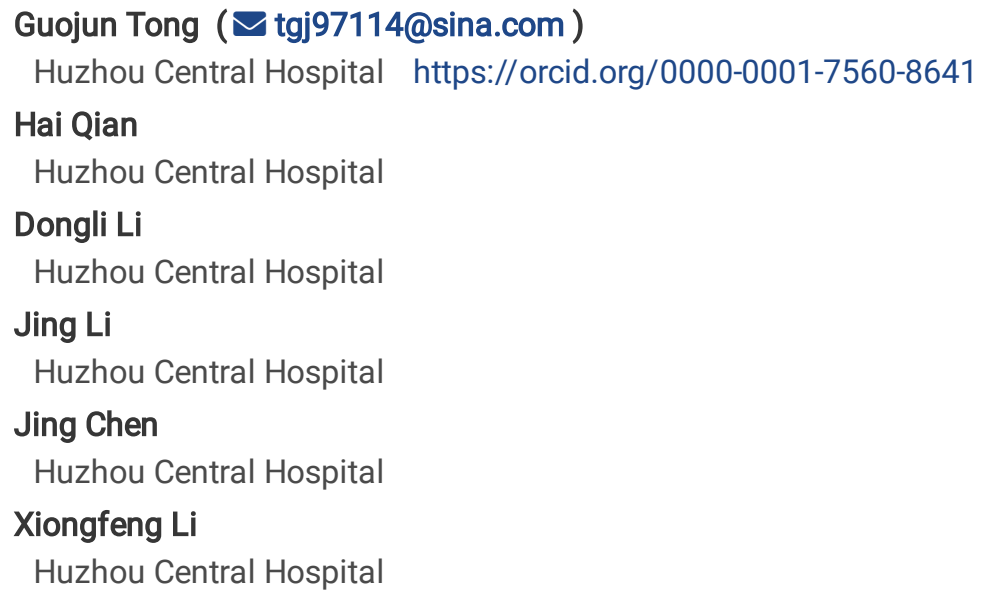




\section{Abstract \\ Background}

In our study, we administered various concentrations of antibiotics to rats, to investigate the changes in the flora of rat feces.

\section{Methods}

Similar Sprague Dawley female rats $(n=84)$ were divided into A - $G$ groups. The rat feces were collected for microbial analysis. The analytical method used a different culture medium for bacterial cultures and count colonies under a microscope. On the 11th and 15th days, we dissected one rat in each group, taking $5 \mathrm{ml}$ of abdominal aortic blood. TNF-a, IL1- $\beta$, IL-6, and C-reactive protein (CRP) were detected by an enzyme-linked immune sorbent assay (ELISA).

\section{Results}

Rats have an average weight of $176.26 \mathrm{~g}$. Between the groups no significant difference was found for starting average weight ( $p>$ 0.05 ) but after experiments, significant differences existed in weight, food intake, water intake, and stool samples within 2 hours ( $p=$ $0.04,0.016,<0.001$, and 0.009 , respectively). Significant differences were found between the groups for nine tested microbiotas ( $p<$ 0.001). TNF-a,IL1- $\beta \square I L-6 \square$ and CRP were significantly different between all groups $(p<0.001)$.

\section{Conclusions}

Antibiotics can cause disorder in rat intestinal flora, also causing an inflammatory response in their blood system.

\section{Background}

Antibiotics first appeared in the 1940s and were regarded as a miracle of modern medicine [1]. The health of human and life expectancy has been improved because of antibiotics [2]. After over a century, antibiotics have developed properties and susceptibility to microorganisms. However, changes to microbial organisms and the struggle to fight microbiota infections, with antibiotics, has not stopped. The intestines are where bacterial flora, beneficial and pathogenic organism gather and in a healthy population, the intestinal flora is balanced. When using antibiotics, this balance is often destroyed leading to intestinal diseases, such as antibiotic associated diarrhea, inflammatory bowel disease (IBD) [3], and pseudomembranous colitis[4].. Diarrhea is a common presentation of intestinal enteritis, with almost 1.7 billion cases of diarrheal disease are reported globally each year [5]. Notably, acute enteritis or diarrheal disease is the second leading cause of death worldwide, accounting for over 1 million deaths annually [6] with higher mortality rates in developing countries [7]. The intestine is a complex organ necessitating sophisticated and comprehensive animal models to study its function and disease [8]. The immune system within the intestine is also complex, combining coordinated responses between the innate and adaptive immune systems within the intestinal mucosa $[9,10]$. The immune system can stimulate cellular and liquid immunity to respond to the intestinal mucosa, resulting in acute inflammation. While changes in the gut microbiota cause an inflammatory response in the intestinal mucosa, causing an inflammatory response in the blood. The main lymphocytic cellular components of the adaptive immune system comprise CD8 + and CD4 + T-cells. Cytotoxic CD $8+T$-cells can also enhance the release of effector cytokines, such as tumor necrosis factor-a (TNF-a) and interleukin-6 (IL-6), which collectively promote macrophage activation and cell death $[8,11]$. Other inflammatory factors and proteins also exhibit high expression, such as interleukin-1 $\beta$ (IL1- $\beta$ ) and Creactive protein (CRP).

Rats are frequently used as an animal model to study intestinal injury and disease. Chemicals used to incite acute inflammation in murine models are also useful in rat models [8]. Sprague Dawley (SD) rats are easily raised and controlled because of their mild temperament. They have been used for the establishment of various disease models, such as skin photoaging, glioma, pancreaticoduodenal transplantation, heart transplantation, and intestinal flora imbalance[12, 13]. For example, the 2,4,6trinitrobenzenesulfonic acid (TNBS) model was initially developed for rats, and is widely used in other organisms $[8,14]$.

Changes to the intestinal microbial organisms can affect intestinal inflammation, studies have shown the addition of probiotic bacteria in diets causes specific bacteria to change the metabolic profile supporting the growth of carbohydrate-reducing bacteria [15, 
16]. Previous studies [17-20] on intestinal dysbiosis patients demonstrated that the dysbiosis signature in Crohn's disease was characterized by five bacterial organisms: an increase in Ruminococcus gnavus, and a decrease in Faecalibacterium prausnitzii, Bifidobacterium adolescentis, Dialister invisus, and an unknown Clostridium cluster XIVa.

In this study, different doses of antibiotics (single and combination) were stomach-fed to rats to show the changes in their intestinal flora and blood inflammatory responses. The target flora included Staphylococcus aureus, Bifidobacterium, yeast, Bacteroides, Clostridium, anaerobic bacteria, E. coli, Enterococcus, and Lactobacillus. The inflammation factors examined were TNF-a, IL1- $\beta$, IL-6, and CRP.

\section{Methods}

\section{Rats}

Sprague Dawley (SD) female rats $(n=84)$, obtained from Liaoning Changsheng Biotechnology Co., Ltd. (Benxi, China) (Experimental Animal Production License No.: SCXK (Liao)), ranged in weight from 172.4 to $179.5 \mathrm{~g}$. Within 24 hours of receipt, the health of the animals was tested, and they were observed before grouping. Only qualified animals were used for the experiments. All animals were introduced to quarantine and adaptive feeding in the specific pathogen free barrier system of this institution for 9 days. Environmental conditions comprised a day and night cycle ( $12 \mathrm{~h}$ light/ $12 \mathrm{~h}$ dark); air exchange times $\geq 15$ times/h; temperature of $20-26^{\circ} \mathrm{C}$; pressure gradient $\geq 10 \mathrm{~Pa}$; relative humidity $40-70 \%$. All procedures were performed under the National Institute of Health Guide for the Care and Use of Laboratory Animals (NIH Publications No. 80-23) revised in 1996. The researchers tried all efforts to minimize the number of animals used.

\section{Experimental drugs, reagents, and instruments}

Drugs used included clindamycin hydrochloride (Shanghai Maclean Biotechnology Co., Ltd., Shanghai, China) with 99\% purity; ampicillin (Hebei Bailingwei Superfine Material Co., Ltd.) with 98\% purity; streptomycin (Tianjin Guangxia Fine Chemicals Institute) with $\geq 90 \%$ purity.

We purchased rat IL1- $\beta$, IL-6, TNF-a, rat CRP from Bioswamp (Wuhan, China). Bacteroides-Bile-Enterprise Agar (BBE), Lactobacillus selective agar, and Dichloran Rose Bengal Chloramphenicol (DRBC) agar were purchased from Qingdao Science and Technology Industrial Park Haibo Zhang Biotechnology Co., Ltd. (Qingdao, China). Anaerobic bacteria agar, Trypticase phytone yeast extract (TPY) agar medium, and mannitol sodium chloride agar medium were purchased from Beijing Luqiao Technology Co., Ltd. (Beijing, China). Eosin Methylene Blue (EMB) agar was purchased from Beijing Road and Bridge Technology Co., Ltd. (Beijing, China). Citrate Azide Tween ${ }^{\circledR}$ Carbonate (CATC) agar was purchased from Qingdao High-Tech Industrial Institute Haibo Zhang Technology Co., Ltd. (Qingdao, China). Reinforced Clostridium Culture Medium was purchased from Beijing Luqiao Rapid Co., Ltd. (Beijing, China).

The electric day constant temperature incubator was purchased from Tianjin Taisite Instrument Co., Ltd. (Tianjin City, China). The Lab systems Multiskan MS micro plate reader was purchased from Thermo Fisher (Pittsburgh, PA, USA). The low-speed condensation centrifuge was purchased from Shanghai Luxiangyi Centrifuge Instrument Co., Ltd. (Shanghai, China). The electronic balance was purchased from Yuyao Jinnuo Tianping Instrument Co., Ltd. (Zhejiang Sheng, China). The upright microscope was purchased from Japan Nikon Guangxuan Microscope Manufacturing Co., Ltd. (Tokyo, Japan).

\section{Animal grouping and drug delivery}

Group A was the control group, group B was the low-dose clindamycin group (250 mg/kg), group C was the middle-dose clindamycin group (500 mg/kg), and group D was the high-dose clindamycin group (750 mg/kg). Group E was the low-dose triple antibiotic group (clindamycin, ampicillin, and streptomycin; $250 \mathrm{mg} / \mathrm{kg}, 272.1 \mathrm{mg} / \mathrm{kg}$, and $136.1 \mathrm{mg} / \mathrm{kg}$, respectively). Group F was the medium-dose triple antibiotic group (clindamycin, ampicillin, and streptomycin; $500 \mathrm{mg} / \mathrm{kg}, 563.7 \mathrm{mg} / \mathrm{kg}$, and $281.8 \mathrm{mg} / \mathrm{kg}$, respectively). Group G was the high-dose triple antibiotic group (clindamycin, ampicillin, and streptomycin; $750 \mathrm{mg} / \mathrm{kg}, 835.8 \mathrm{mg} / \mathrm{kg}$, and $417.9 \mathrm{mg} / \mathrm{kg}$, respectively). The experiment was divided into two stages: the modeling period (days $1-7$ ) and the recovery period (days 8 - 15). The administration volume was $10 \mathrm{ml} / \mathrm{kg}$ once a day through stomach feeding by oral needle during the modeling period between 8:30 10:00 AM. The intragastric administration was stopped during the recovery period.

\section{Collected and analyzed indicators}

Page $3 / 20$ 
The weight, food intake volume, water intake volume, and stool samples taken on the $1^{\text {st }}, 3^{\text {rd }}, 5^{\text {th }}, 7^{\text {th }}, 9^{\text {th }}, 11^{\text {th }}$, and $14^{\text {th }}$ days within 2 hours were collected. For each rat, the fecal microbial flora on the $1^{\text {st }}, 4^{\text {th }}, 8^{\text {th }}, 11^{\text {th }}$, and $14^{\text {th }}$ days were examined. The microbial organisms detected were Staphylococcus aureus, Bifidobacterium, yeast, Bacteroides, Clostridium, anaerobic bacteria, E. coli, Enterococcus, and Lactobacillus. Five milliliters of abdominal aortic blood of SD rats were collected. The TNF-a, IL1- $\beta$, IL-6, and CRP was detected in the blood serum without diluting by an enzyme-linked immune sorbent assay (ELISA).

\section{Experimental protocol}

We Mixed $1 \mathrm{~g}$ of feces with $9 \mathrm{ml}$ of tryptone soy broth, diluted to the appropriate concentration, and took $20 \mu \mathrm{l}$ of the sample and spread it evenly on the agar medium using a coating bar. Organisms were plated on to mannitol sodium chloride agar medium plates, $\mathrm{EMB}$, and CATC agar plates under aerobic conditions at $37^{\circ} \mathrm{C}$ for 48 hours. Organisms cultured on TPY agar medium plates, BBE agar plates, reinforced Clostridium medium plates, anaerobic agar plates, and Lactobacillus selective agar plates were cultured at $37^{\circ} \mathrm{C}$ for 48 hours in anaerobic conditions. Organisms inoculated on DRBC agar plates were cultured for 5 days at $28^{\circ} \mathrm{C}$ in aerobic conditions. Colonies were enumerated using equation 1.

number of coloniies $(\mathrm{CFU} / \mathrm{g})=$ number of plate colonies $\times 50 \times$ dilution factor

(1)

where the dilution factor was $10^{6}(\mathrm{E} 6)$ as a uniform unit.

Half of the rats were dissected on the $11^{\text {th }}$ day with the other half were dissected on the $15^{\text {th }}$ day. The rats were anesthetized with $2 \%$ pentobarbital sodium $(0.2 \mathrm{ml} / 100 \mathrm{~g})$ by intra peritoneal injection and dissected by abdominal incision. Under anesthesia, SD rats were euthanized by draining abdominal aorta blood. Five milliliters abdominal aortic blood was collected for inflammation factors detection. TNF-a, IL-1 $\beta$, IL-6, and CRP was detected in the blood serum without diluting, by an ELISA.

\section{Ethics approval and consent to participate}

This study follows the Basel Declaration 2010. Most authors of this article have been trained in animal experiments and have obtained a certificate of competency. We use animals to a minimum in terms of animal welfare principles without affecting the accuracy of the experiment. The commissioned experimental unit has qualifications and right to use animals, approved by Institution Animal Care and Use Committee (IACUC) of Xi'an United Nations Quality Detection Technology CO., Ltd. All applicable international, national, and/or institutional guidelines for care and use of animals were followed.

\section{Statistical analysis}

SPSS 21 software (IL, USA) was used to analyze all data, using ANOVA and F-test to measure and analyze the data. Weighting methods and crosstabs were also used for data analysis. Categorical variables were used by crosstabs and chi-square test. The independent sample T-test and LSD method were used for comparative analysis between two sets of measured variables. $P<0.05$ was as statistic difference.

\section{Results}

\section{Comparison of general indicators between groups}

All rats were successfully tested, and the resultant data was complete and accurate (Figure $1 \mathrm{~A}$ ). The average starting weight of all rats before the experiment was $17.26 \pm 2.49 \mathrm{~g}$, and there were no significant differences between groups $\mathrm{A}-\mathrm{G}(p<0.05)$. There were significant differences in groups A (control) to $\mathrm{G}$ (treated) for weight, food intake, water intake, and stool samples 2 hours after antibiotic use $(p=0.04,0.016,<0.01$, and 0.009 , respectively). Means and standard deviations are shown in Table 1 and Figure 1 (B E).

\section{Comparison of total microbiota between groups}

Nine intestinal floral (Staphylococcus aureus, Bifidobacterium, yeast, Bacteroides, Clostridium, anaerobic bacteria, E. coli, Enterococcus, and Lactobacillus) were cultured using a special medium [Mannitol sodium chloride agar medium (culture of Staphylococcus aureus); TPY agar medium (culture of Bifidobacterium); DRBC agar (cultured yeast); Bacteroides-bile-escin agar (BBE) (culture of Bacteroides); 
reinforced cloaca culture medium (culture of Clostridium); anaerobic agar (culture of anaerobic bacteria); Eosin blue agar medium (EMB) (culture of E. coli); CATC agar (cultured Enterococcus); lactic acid Bacillus selective agar (cultured Lactobacillus)] and counted with an upright microscope (Ni-U,Japan) (Figure 2 and 3 ). Microbial loads for these nine organisms at the $1^{\text {st }}, 4^{\text {th }}, 8^{\text {th }}, 11^{\text {th }}$ and $14^{\text {th }}$ days for each animal group were compared. The means, standard deviations, and $95 \%$ confidence interval $(\mathrm{Cl})$ of each group are: (A): 1,376.7 \pm 3,683.8 (95\% Cl: 562.15 - 2,191.26); (B): $687.06 \pm$ 1,498.74 (95\% Cl: 355.65 - 1,018.45); (C): 1,474.89 $\pm 4,187.53$ (95\% Cl: 548.96 - 2,400.83); (D): $478.17 \pm$ 1,758.11 (95\% Cl: 65.03 - 891.31); (E): $664.50 \pm 1,567.91$ (95\% Cl: 317.80 - 1,011.19); (F): $403.77 \pm$ 1,171.99 (95\% Cl: 144.62 - 662.92); (G): 3,609.76 \pm 21,206.52 (95\% Cl: -1079.38 \pm 8,298.91) (CFU/g). There were no significant difference between groups $A / B, A / C, A / D, A / E, A / F$, and $A / G$ comparing the total nine bacterial organisms on all collected days, whereas significant differences existed between groups $G / B, G / D, G / E$, and $G / F$ after least significant differences (LSD) analysis. There were significant differences between groups $(F=1.432, p<0.001)$. Details are shown in Table 2 and Figure $4(A)$.

The microbial loads for all nine organisms were compared in groups A - $\mathrm{G}$ at each day, using before and after weighted methods. All comparisons between groups were significantly different $(p<0.001)$ (Table 3 and Figure 4(B - C)).

\section{Comparison of microbiota on different days between groups}

Further analysis was conducted because no significant differences in the nine microbial load existed between groups A (Control) and B

- G. Therefore, we compared the nine microbial loads and the load of each microbiota on the $1^{\text {st }}, 4^{\text {th }}, 8^{\text {th }}, 11^{\text {th }}$, and $14^{\text {th }}$ day in each group. On the $1^{\text {st }}$ day, there were significant differences in total nine microbiota between groups $A$ and $B-G(p<0.001)$. On the $4^{\text {th }}$ day, there were significant differences in the nine microbiota between groups $A$ and $B-G(p<0.001)$. On the $8^{\text {th }}$ day there were significant differences in the nine microbial loads between groups $A$ and $B-G(p=0.015,0.006,0.002,0.001,0.001$, and 0.001 , respectively). But on the $11^{\text {th }}$ day there were no significant differences in the nine microbial loads between groups $A$ and $B-G(p=0.687,0.353,0.537$, $0.989,0.070$, and 0.515 , respectively). There were also no significant differences in the nine microbial loads on the $14^{\text {th }}$ day between groups A and B - $\mathrm{G}(p=0.680,0.223,0.250,0.816,0.69$, and 0.472 , respectively). These changes show that the intestinal flora gradually recovered after drugs were stopped. While there were significant differences in the loads of each microbial organism between all groups at all involved days, following weighted analysis $(p<0.001)$ (Table 4 and Figure 4(D \& E)).

\section{Comparison of inflammation factors between groups}

There were significant differences in the levels of TNF-a between groups A and B - $\mathrm{G}(p=0.021,0.048,0.012,0.046,0.025$, and 0.012 , respectively) with ANOVA showing a significant difference within groups $(F=2.343, p<0.001)$. IL $1-\beta$ had significant differences between groups $\mathrm{A}$ and $\mathrm{B}-\mathrm{G}(p=0.042,0.033,0.020,0.047,0.022$, and 0.015 , respectively $)$ with ANOVA showing $\mathrm{F}=1.186, P<0.001$. Significant difference existed between all groups comparing IL-6 with $\mathrm{A}(\mathrm{B} / \mathrm{A}, p=0.012 ; \mathrm{C} / \mathrm{A}, p=0.025 ; \mathrm{D} / \mathrm{A}, p=0.047 ; \mathrm{E} / \mathrm{A}, p=0.022$; $\mathrm{F} / \mathrm{A}, p=0.015 ; \mathrm{G} / \mathrm{A}, p=0.001$ ); ANOVA analysis showed $\mathrm{F}=1.337, p<0.001$. There were significant differences in CRP levels between groups $A$ and other groups (B/A, $p=0.023 ; \mathrm{C} / \mathrm{A}, p=0.042 ; \mathrm{D} / \mathrm{A}, p<0.001 ; \mathrm{E} / \mathrm{A}, p<0.001 ; \mathrm{F} / \mathrm{A}, p=0.001 ; \mathrm{G} / \mathrm{A}, p<0.001)$ and ANOVA showed $\mathrm{F}=2.807, p<0.001$. Mean values, standard deviations, and $95 \% \mathrm{Cl}$ are shown in Table 5 and Figure 5 .

\section{Discussion}

Clinically, we have witnessed an increase in the incidence of intestinal dysfunction. Medical history has revealed a variety of reasons, such as bad living habits, irregular work, and long-term use of antibiotics. Antibiotics are an indispensable drug in modern medicine, but proper use of these medications and standardized medical procedures have always been demanded of most physicians. The gradual promotion of Enhanced Recovery After Surgery (ERAS) [21-24] has reduced the clinical application of antibiotics, intestinal complications, and other complications caused by double infections. This study used rats to explore the effects of antibiotics on intestinal flora, focusing on nine common microbial organisms, both beneficial and pathogenic. An alternate rat model of acute inflammation had also been established using the biological incitant Campylobacter jejuni, suggesting that both chemical and biological agents were useful to induce colitis in the rat animal model [25]. Researchers have also utilized the rat model to investigate the impact of fiber-rich diets on intestinal microbial community structure [26, 27]. Based on these publications, we chose the rat as a model for our research on intestinal flora following antibiotic use. This study showed that the body weight of the rats changed after antibiotic molding, suggesting that antibiotics influence the nutritional status of the rat. This may be because glycolipid disorders are closely related to intestinal flora and its metabolites [28, 29]. Here, food intake decreased and water intake increased significantly with an increase in antibiotic dose, and there were changes for defecation dependent on dose administration within 2 hours after antibiotic use. These changes indicate intestinal dysfunction, which led us to explore the changes in the intestinal micro-ecology.

Page 5/20 
The host intestinal tract contains a diverse community of bacteria, totaling $10^{13}-10^{14}$ bacterial cells [30], with organisms most often belonging to the Bacteroidetes, Firmicutes, Actinobacteria, Spirochete, and Proteobacteria phyla [8, 31, 32]. Here, the nine target microbial organisms studied are indicated at $10^{6}$ (CFU/g). Homeostatic interactions between the host and the resident microbiota occur in the intestine, and changes in microbial abundance might lead to intestinal inflammation [32]. Investigation has well documented that commensal microbiota are important in maintaining a healthy intestine by preventing the overgrowth of pathogenic microorganisms and help regulate and maintaining a quiescent intestinal immune system [33]. Once the healthy flora is destroyed and the intestinal flora is disordered, damage to the intestinal mucosa can be caused by changes in the immune system through the braingut axis, the intestinal-bacteria axis, and the abnormal flora metabolites. Reports indicated that developing aberrant immune responses could occur from increased exposure to the commensal microbiota [31, 34]. Attack of the intestinal mucosa by immune cells can produce intestinal diseases. Studies by Hoffmann $\mathrm{C}$ and Sherman MA reported that modifications to the community structure of the intestinal microbiota could incite disease, often by the uncoordinated expression of pro-inflammatory cytokine profiles with the simultaneous loss of anti-inflammatory signaling [31,35]. IBD is an intestinal inflammatory condition affecting over two million people in the United States [36]. Intestinal dysbiosis for patients with IBD has shown a characteristic pattern of a decrease in commensal microbial diversity, with most of the decrease in Firmicutes and Bacteroides in the intestinal microflora which are the two most abundant groups in the normal flora [17-19].Staphylococcus aureus is a major human pathogen that causes a wide range of clinical infections. It is a leading cause of bacteremia and infective endocarditis and osteoarticular, skin and soft tissue, pleuropulmonary, and device-related infections [37]. This bacterium can reside in, and infect a wide range of host tissues, from superficial surfaces like the skin to deeper tissues like in the gastrointestinal tract, heart and bones [38]. S. aureus infectious capacity and its success as a pathogen is associated with the expression of virulence factors, among which the production of a wide variety of toxins has been highlighted [39]. Thus, in this study, Staphylococcus aureus was tested and analyzed. It increased compared with the control group, while as the dose of the antibiotic increased, the increase in bacterial load was not obvious, indicating that the drug inhibited the strain. We also analyzed $S$. aureus load chronologically throughout the tested days, finding it was highest on day 14 . The rebound phenomenon was obvious after stopping the drug. This might be related to the strong regeneration ability after the drug being excreted while the mechanisms are still unknown.

Bifidobacterium are defined as a group of living microorganism supplements, which confer health benefits on the host when administered in adequate amounts [40]. When the body lacks a certain amount of Bifidobacterium, it will cause diseases [41, 42]. Here, the beneficial bacterium was significantly reduced in groups B, D, E, and F than the control group, however it was elevated in the middle-dose monotherapy group $(C)$ and the high-dose combination group $(G)$. We considered that the middle single dose and high combined dose groups might have an obvious rebound effect after stopping the drug. This was also confirmed by comparison on different days.

Yeast cells are often used in industrial fermentation processes for their ability to efficiently convert relatively high concentrations of sugars into ethanol and carbon dioxide [43]. Previous literature has shown how nitrogen overflow by yeast benefits $L$. plantarum in grape juice and contributes to the emergence of mutualism with L. lactis in a medium with lactose [44]. Yeast is not a common intestinal microbiota, and it is absent in the control group. It was only found in the low- and middle-doses of the triple-agent groups ( $E$ and F), and the amount present was not larger than the other organisms. We believed this was the colonization of infrequent flora and was one manifestation of intestinal flora disorder.

Bacteroide is a gram-negative, non-spore, and obligate anaerobic Bacillus. This study showed a significant reduction in the high-dose medication group, indicating that it was sensitive to high-dose and combination drugs, and the rebound effect was very obvious after stopping the drug. A previous study has shown that its metabolic disorders are associated with type 2 diabetes [45]. Obesity was related to the intestinal flora at the phylum level, with the dominant phylum of the intestinal flora in obese people changed to Actinobacteria and Firmicutes, and in lean people changed to Bacteroidetes [46].

Clostridium organisms are anaerobic, gram-positive, rod-shaped, and endospore-forming bacteria belonging to the phylum Firmicutes, constituting both a class and genus in the phylum [47]. Infection of the colon with the gram-positive bacterium Clostridium difficile is potentially life threatening, especially in elderly people and patients who have dysbiosis of the gut microbiota, following antimicrobial drug exposure. $C$. difficile is the leading cause of healthcare associated infective diarrhea [48]. This study showed that compared with the control group, Clostridium decreased in the single-agent group whereas it increased in the combined drug group, revealing this organism had strong regeneration after the drug stopped, confirmed by Table 4 and Fig. 4(E). This finding can provide a reference to treat clinical Clostridium infection. Another study also provides a guideline to treat Clostridium infections [49]. 
Anaerobic bacteria have pivotal roles in the microbiota of humans, and they are significant infectious agents involved in several pathological processes, especially in immunocompromised individuals. Their isolation, cultivation, and correct identification differs significantly from the workup of aerobic organisms, although using innovative technologies changes anaerobic diagnostics dramatically [50]. Here, anaerobic bacteria were significantly decreased in all other groups during drug feeding periods than the control group. However, during the recovery period, this organism rebound significantly, which was confirmed on different analysis days.

Although Escherichia coli can be an innocuous resident of the gastrointestinal tract, it also has the pathogenic capacity to cause significant diarrheal and extraintestinal diseases. Pathogenic variants of $E$. coli can cause much morbidity and mortality worldwide [51]. E. coli has been regarded as a component of the normal intestinal flora for a long time and is a non-pathogenic bacterium[52]. It was not until the middle of the 20th century it was recognized that some serotypes of $E$. coli were pathogenic to humans and animals[53]. Pathogenic $E$. coli can cause a broad range of human diseases that span from the gastrointestinal tract to extraintestinal sites such as the urinary tract, bloodstream, and central nervous system [54,55]. Here, we found $E$. coli was killed on the 1 st and 4 th days, but the rebound phenomenon was obvious after stopping the drug.

Enterococcus is a gram-positive coccus, which is widely distributed in the natural environment and in the digestive tract of humans and animals in a maintained balance. Enterococcus strains that adhere strongly to the intestinal epithelium, form biofilms, and possess antioxidant defense mechanisms, seem to have the greatest influence on the inflammatory process [56]. Here, a significant increase in this organism in groups of different doses of single and combination drugs at all tested days was observed. We found it decreased significantly on the $1 \mathrm{st}$ and 4 th days, but rebounded significantly after drugs were stopped, which means both single and combined drug use affected this organism independent on dose.

Lactobacillus comprises 173 genera, with many genomes available to study taxonomy and evolutionary events, reported by a previous study [57]. As a probiotic, Lactobacillus was significantly reduced in groups B - F than the control group on the 4th, 8 th, and 11 th days. We were also skeptical of the significant increase in the high-dose combination group (G) as Table 4 shows it was 10 times higher in group $\mathrm{G}$ than the control group on the 1st day, likely for not killing it at the beginning. We found that Lactobacillus peaked on the 1st day and did not rebound significantly after stopping the drug, Fig. 4D. This proved that the drug killed Lactobacillus by continuous administration.

Here, we found that the nine target intestinal microorganisms were killed to some extent after drug administration, and rebounded to varying degrees after drug withdrawal, especially on the 11th and 14th days (Fig. 4E), but the laws of drug administration and dosage were not obvious, and irregular disorders appeared.

Intestinal microecological disorders can also affect the immune system. This information is presented to T-cell populations through the secretion of cytokines to facilitate cell maturation and proliferation [58]. This study found that TNF- $a$ and IL- 6 were significantly higher in experimental groups than in the control group, and there was a significant relationship with the dose administered.

Inflammation induces IL1- $\beta$ production in Kupffer cells and hepatocytes [59]. In this study, the IL1- $\beta$ was significantly greater in dose administered group than the control group. Lee et. al. analyzed the whole genome sequence database of the Atlantic salmon (Salmo salar) and identified five CRP/serum amyloid P-component (SAP) like molecules, CRP/SAP-1a, CRP/SAP-1b, CRP/SAP-1c, CRP/SAP-2, and CRP/SAP-3 [60]. CRP is a novel topic for studying inflammation and related diseases [61-63], being associated with chronic inflammation; thus, here CRP was examined in the abdominal blood of the SD rats. The results showed that elevated CRP was associated with groups receiving antibiotics, as with the discussed inflammatory factors. The four inflammation factors increased depending on the drug dose, which showed the increase of the single and combined dose, leading to an increase of intestinal flora disturbance, with the four target inflammatory factors in serum increasing significantly in the humoral and cellular immune mechanisms (Fig. 5).

However, there are shortcomings in this manuscript. We did not explore the mechanisms between intestinal flora disorder and the rat intestinal inflammation. Also, we did not genetically analyze the gut target flora.

\section{Conclusions}

Antibiotics can cause disorder in the intestinal target flora organism of rats, without apparent law of the doses and combination of antibiotic use. Antibiotics can also causes an inflammatory response in the blood system of rats. The current research can provide a basis for intestinal inflammatory diseases caused by intestinal microbial disorders.

Page 7/20 


\section{Abbreviations}

\begin{tabular}{|c|c|}
\hline BBE & Bacteroides-Bile-Enterprise \\
\hline $\mathrm{Cl}$ & Confidence interval \\
\hline CRP & C-reactive protein \\
\hline DRBC & Dichloran Rose Bengal Chloramphenicol \\
\hline ELISA & Enzyme-linked immune sorbent assay \\
\hline EMB & Eosin Methylene Blue \\
\hline ERAS & Enhanced Recovery After Surgery \\
\hline IACUC & Institution Animal Care and Use Committee \\
\hline IBD & Inflammatory bowel disease \\
\hline LSD & Least significant differences \\
\hline SD & Sprague Dawley \\
\hline TPY & Trypticase phytone yeast \\
\hline ANOVA & Analysis of variance \\
\hline CATC & Citrate Azide Tween $®$ Carbonate \\
\hline TNBS & 2,4,6-trinitrobenzenesulfonic acid model \\
\hline TNF-a & Tumor necrosis factor-a \\
\hline IL-6 & Interleukin-6 \\
\hline IL $1-\beta$ & Interleukin-1 $\beta$ \\
\hline SAP & Serum amyloid P-component \\
\hline
\end{tabular}

\section{Declarations}

\section{Ethics approval and consent to participate}

This study follows the Basel Declaration 2010. Most authors of this article have been trained in animal experiments and have obtained a certificate of competency. We use animals to a minimum in terms of animal welfare principles and without affecting the accuracy of the experiment. Xi'an United Nations Quality Detection Technology Laboratory was commissioned to perform our experiments under his IACUC permission. All applicable international, national, and/or institutional guidelines for care and use of animals were followed.

\section{Consent for publication}

Not applicable.

\section{Availability of data and material}

The datasets used and/or analyzed during the current study are available from the corresponding author.

\section{Competing interests}


The contents of this manuscript have not been copyrighted or published. There are no related manuscripts or abstracts, published or unpublished, by any authors of this manuscript. The authors indicated no conflicts of interests.

\section{Funding}

This study was supported by Project 2018C37090 to Guojun Tong. The fund is mainly used for experiment, data collection, experimental index detection, statistical analysis, and experts communication.

\section{Author contribution}

All authors discussed the methodology and considered it available. GT and $\mathrm{HQ}$ analyzed all experimental data. XL, DL, JL, and JC reviewed the statistical results and participated in all figures, drawings, and stitching with GT. GT authored the paper. The research teams of Xi'an United Nations Quality Detection Technology Laboratory performed the experiments. All authors reviewed the manuscript and approved the manuscript.

\section{Acknowledgments}

We thank Professor Licheng Dai, Liqin Li, researcher Yingrong Chen, Zhihong Ma (Central Laboratory, Huzhou Central Hospital) who gave us many suggestions.

\section{References}

[1].GUO Kang-xiao,YIN Kang-kang, WANG Huan, et al. The effect of modeling dysbacterial diarrhea with antibiotics on molecular

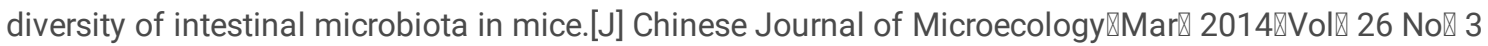

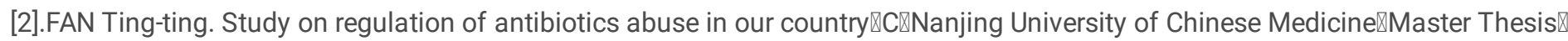
2012区

[3].Jonathan P, Jacobs, Maryam Goudarzi, Namita Singh, et al. A Disease-Associated Microbial and Metabolomics State in Relatives of Pediatric Inflammatory Bowel Disease Patients.[J] Cell Mol Gastroenterol Hepatol 2016;2:750-766;

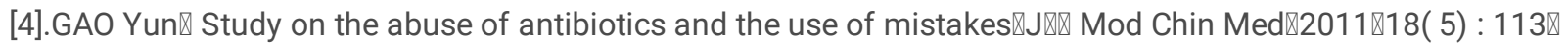

[5].World Health Organization. Diarrheal disease. In: Fact Sheet Number 330. 2013.

http://www.who.int/mediacentre/factsheets/fs330/en/.

[6].Ahmed SM, Hall AJ, Robinson AE, et al. Global prevalence of noro-virus in cases of gastroenteritis: a systematic review and metaanalysis. Lancet Infect Dis. 2014;14(8):725-30.

[7]. Liu L, Johnson HL, Cousens S, et al. Global, regional, and national causes of child mortality: an updated systematic analysis for 2010 with time trends since 2000. Lancet. 2012;379(9832):2151-61.

[8].Janelle A. Jiminez, Trina C. Uwiera, G. Douglas Inglis, Richard R. E. Uwiera. Animal models to study acute and chronic intestinal inflammation in mammals.[J]Gut Pathog (2015) 7:29.

[9].Wallace KL, Zheng LB, Kanazawa Y, Shih DQ. Immunopathology of inflammatory bowel disease. World J Gastroenterol. 2014;20(1):6-21.

[10].Geremia A, Biancheri P, Allan P, Corazza GR, Di Sabatino A. Innate and adaptive immunity in inflammatory bowel disease. Autoimmun Rev. 2014;13(1):3-10.

[11].Janeway CAJ, Travers P, Walport M, Sclomchik MJ. Immunobiology: the immune system in health and disease. 5th ed. New York: Garland Science;2001.

[12].Geiger BM, Gras-Miralles B, Ziogas DC,et al. Intestinal upregulation of melanin-concentrating hormone in TNBS-induced enterocolitis in adult zebrafish. PLoS One.2013;8(12):e83194 
[13].Fitzpatrick LR, Meirelles K, Small JS, Puleo FJ, Koltun WA, Cooney RN. A new model of chronic hapten-induced colitis in young rats. J Pediatr Gastroenterol Nutr. 2010;50(3):240-50.

[14]. Krimi RB, Kotelevets L, Dubuquoy L,et al. Resistin-like molecule beta regulates intestinal mucous secretion and curtails TNBSinduced colitis in mice. Inflamm Bowel Dis.2008;14(7):931-41.

[15].Barthel M, Hapfelmeier S, Quintanilla-Martinez L,et al. Pretreatment of mice with streptomycin provides a Salmonella enterica serovar Typhimurium colitis model that allows analysis of both pathogen and host. Infect Immun. 2003;71(5):2839-58.

[16].Gourbeyre P, Denery S, Bodinier M. Probiotics, prebiotics, and synbiotics: impact on the gut immune system and allergic reactions. J Leukoc Biol. 2011;89(5):685-95.

[17]. Hedin C, McCarthy N, Louis P, et al. Altered intestinal microbiota and blood T cell phenotype are shared by patients with Crohn's disease and their unaffected siblings. Gut. 2014; 63(10):1578-86.

[18].Nguyen GC. Bugs and Drugs: Insights into the Pathogenesis of Inflammatory Bowel Disease. American Journal of Gastroenterology. 2011; 106:2143-5.

[19].Sokol H, Lay C, Seksik P, et al. Analysis of bacterial bowel communities of IBD patients: what has it revealed? Inflammatory Bowel Diseases. 2008; 14(6):858-67.

[20].Joossens $M$, Huys $G$, Cnockaert M, et al. Dysbiosis of the faecal microbiota in patients with Crohn's disease and their unaffected relatives. Gut. 2011; 60:631-7.

[21].Chelsia Gillis, Marlyn Gill, Nancy Marlett, et al.Patients as partners in Enhanced Recovery After Surgery: A qualitative patient-led study.BMJ Open. 2017; 7(6): e017002.Published online 2017 Jun 24.doi:10.1136/bmjopen-2017-017002

[22].W. R. Spanjersberg, J. D. P. van Sambeeck, A. Bremers, C. Rosman, C. J. H. M. van Laarhoven. Systematic review and meta-analysis for laparoscopic versus open colon surgery with or without an ERAS programme.Surg Endosc.2015; 29(12): 3443-3453. Published online 2015 Mar 24.doi:10.1007/s00464-015-4148-3

[23]. Leah M. Gramlich, Caroline E. Sheppard, Tracy Wasylak, et al. Implementation of Enhanced Recovery After Surgery: a strategy to transform surgical care across a health system.Implement Sci.2017;12:67. Published online 2017 May 19.doi:10.1186/s13012-0170597-5

[24].Ester Miralpeix, Alpa M. Nick, Larissa A. Meyer, et al. A Call for New Standard of Care in Perioperative Gynecologic Oncology Practice: Impact of Enhanced Recovery After Surgery (ERAS) Programs.Gynecol Oncol. 2016 May; 141(2):371-378.Published online 2016 Mar 9.doi:10.1016/j.ygyno.2016.02.019

[25]. Morales W, Pimentel M, Hwang L, et al. Acute and chronic histological changes of the small bowel secondary to $C$. jejuni infection in a rat model for post-infectious IBS. Dig Dis Sci.2011;56(9):2575-84. doi:10.1007/s10620-011-1662-6.

[26]. Abnous K, Brooks SP, Kwan J, et al. Diets enriched in oat bran or wheat bran temporally and differentially alter the composition of the fecal community of rats. J Nutr. 2009;139(11):2024-31. doi:10.3945/jn.109.109470

[27]. Kalmokoff M, Zwicker B, O'Hara M, et al. Temporal change in the gut community of rats fed high amylose cornstarch is driven by endogenous urea rather than strictly on carbohydrate.availability. J Appl Microbiol. 2013;114(5):1516-28. doi:10.1111/jam.12157.

[28].Sha Di, Yitian Wang, Lin Han, Qi Bao, Zezheng Gao, Qing Wang, Yingying Yang, Linhua Zhao, Xiaolin Tong. The Intervention Effect of Traditional Chinese Medicine on the Intestinal Flora and Its Metabolites in Glycolipid Metabolic Disorders. Evid Based Complement Alternat Med.2019;2019: 2958920.Published online 2019 Jun 4.doi:10.1155/2019/2958920

[29].Lipid metabolism group. Expert consensus on the prevention and treatment of type 2 diabetes mellitus with dyslipidemia in China (revised version in 2017) Chinese Journal of Endocrinology and Metabolism. 2017;33(11)

[30]. Gill SR, Pop M, Deboy RT, et al. Metagenomic analysis of the human distal gut microbiome. Science. 2006;312(5778):1355-9.

doi:10.1126/science.1124234.

Page $10 / 20$ 
[31].Hoffmann C, Hill DA, Minkah N, et al. Communitywide response of the gut microbiota to enteropathogenic Citrobacter rodentium infection revealed by deep sequencing. Infect Immun. 2009;77(10):4668-78. doi:10.1128/IAI.00493-09.

[32].Shen XJ, Rawls JF, Randall T, et al. Molecular characterization of mucosal adherent bacteria and associations with colorectal adenomas. Gut Microbes. 2010;1(3):138-47.doi:10.4161/gmic.1.3.12360

[33].Hawrelak JA, Myers SP. The causes of intestinal dysbiosis: a review. Altern Med Rev. 2004;9(2):180-97.

[34].Tabas I, Glass CK. Anti-inflammatory therapy in chronic disease: challenges and opportunities. Science. 2013;339(6116):166-72. doi:10.1126/ science. 1230720

[35].Sherman MA, Kalman D. Initiation and resolution of mucosal inflammation. Immunol Res. 2004;29(1-3):241-52. doi:10.1385/ IR:29:1-3:241.

[36].Arianna K. DeGruttola, B.S., Daren Low, Ph.D., Atsushi Mizoguchi, M.D., Ph.D.2, and Emiko Mizoguchi, M.D., Ph.D.1,2.Current understanding of dysbiosis in disease in human and animal models.Inflamm Bowel Dis. 2016 May; 22(5): 1137-1150.

doi:10.1097/MIB.0000000000000750

[37]. Steven Y. C. Tong, Joshua S. Davis, Emily Eichenberger, Thomas L. Holland, Vance G. Fowler, Jr.Staphylococcus aureus Infections: Epidemiology, Pathophysiology, Clinical Manifestations, and Management. Clin Microbiol Rev. 2015 Jul;28(3): 603-661.Published online 2015 May 27.doi:10.1128/CMR.00134-14

[38].Divya Balasubramanian, Lamia Harper, Bo Shopsin, Victor J. Torres. Staphylococcus aureus pathogenesis in diverse host environments. Pathog Dis. 2017 Feb;75(1): ftx005. Published online 2017 Jan 19.doi:10.1093/femspd/ftx005

[39]. Diana Oliveira, Anabela Borges, Manuel Simões. Staphylococcus aureus Toxins and Their Molecular Activity in Infectious Diseases. Toxins (Basel) 2018 Jun; 10(6): 252.Published online 2018 Jun 19. doi: 10.3390/toxins10060252.

[40]. Kim BJ, Park T, Moon HC, et al. Cytoprotective alginate/polydopamine core/shell microcapsules in microbial encapsulation. Angew Chem Int Ed Engl.2014;53(52):14443-14446.

[41]. Mei L, He F, Zhou RQ, et al. Novel intestinal-targeted Ca-alginate-based carrier for pH-responsive protection and release of lactic acid bacteria.ACS Appl Mater Interfaces.2014;6(8):5962-5970.

[42]. D'Orazio G, Di Gennaro P, Boccarusso M, et al. Microencapsulation of new probiotic formulations for gastrointestinal delivery in vitro study to assess viability and biological properties. Appl Microbiol Biotechnol.2015;99(22):9779-9789.

[43]. Maria C Dzialo, Rahel Park, Jan Steensels, Bart Lievens, Kevin J Verstrepen. Physiology, ecology and industrial applications of aroma formation in yeast.FEMS Microbiol Rev. 2017 Aug; 41(Suppl 1): S95-S128. Published online 2017 Aug

17.doi:10.1093/femsre/fux031

[44]. Olga Ponomarova, Natalia Gabrielli, Daniel C. Sévin, Michael Mülleder, Katharina Zirngibl, Katsiaryna Bulyha, Sergej Andrejev, Eleni Kafkia, Athanasios Typas, Uwe Sauer, Markus Ralser, Kiran Raosaheb Patil. Yeast Creates a Niche for Symbiotic Lactic Acid Bacteria through Nitrogen Overflow. Cell Syst.2017 Oct 25;5(4): 345-357.e6.doi:10.1016/j.cels.2017.09.002

[45]. Masanori Horie, Takamasa Miura, Satomi Hirakata, Akira Hosoyama, Sakiko Sugino, Aya Umeno, Kazutoshi Murotomi, Yasukazu Yoshida, Taisuke Koike. Comparative analysis of the intestinal flora in type 2 diabetes and nondiabetic mice. Exp Anim. 2017;66(4): 405-416. Published online 2017 Jul 12. doi: 10.1538/expanim.17-0021

[46]. Turnbaugh P.J., Hamady M., Yatsunenko T., Cantarel B.L., Duncan A., Ley R.E., Sogin M.L., Jones W.J., Roe B.A., Affourtit J.P., Egholm M., Henrissat B., Heath A.C., Knight R., Gordon J.I. 2009. A core gut microbiome in obese and lean twins. Nature 457: 480-484. doi: $10.1038 /$ nature07540

[47]. Paredes CJ, Alsaker KV, Papoutsakis ET. 2005.A comparative genomic view of clostridial sporulation and physiology.Nat Rev Microbiol 3:969-978. doi:10.1038/nrmicro1288. 
[48]. Wiep Klaas Smits, Dena Lyras, D. Borden Lacy, Mark H. Wilcox, Ed J. Kuijper. Clostridium difficile infection. Nat Rev Dis Primers. Author manuscript; available in PMC 2017 Jun 1.

[49]. L Clifford McDonald, Dale N Gerding, Stuart Johnson, Johan S Bakken, Karen C Carroll, Susan E Coffin, Erik R Dubberke, Kevin W Garey, Carolyn V Gould, Ciaran Kelly, Vivian Loo, Julia Shaklee Sammons, Thomas J Sandora, Mark H Wilcox. Clinical Practice Guidelines for Clostridium difficile Infection in Adults and Children: 2017 Update by the Infectious Diseases Society of America (IDSA) and Society for Healthcare Epidemiology of America (SHEA). Clin Infect Dis.2018 Apr 1;66(7): e1-e48.Published online 2018 Feb 15.doi:10.1093/cid/cix1085

[50]. Márió Gajdács, Gabriella Spengler, Edit Urbán. Identification and Antimicrobial Susceptibility Testing of Anaerobic Bacteria: Rubik's Cube of Clinical Microbiology?. Antibiotics(Basel) 2017 Dec;6(4): 25.Published online 2017 Nov 7.doi:10.3390/antibiotics6040025

[51]. Matthew A. Croxen, Robyn J. Law, Roland Scholz, Kristie M. Keeney, Marta Wlodarska, B. Brett Finlay. Recent Advances in Understanding Enteric Pathogenic Escherichia coli. Clin Microbiol Rev.2013 Oct;26(4): 822-880.doi:10.1128/CMR.00022-13

[52] Zachary R. Stromberg, James R. Johnson, John M. Fairbrother, Jacquelyn Kilbourne, Angelica Van Goor, Roy Curtiss, 3rd, Melha Mellata. Evaluation of Escherichia coli isolates from healthy chickens to determine their potential risk to poultry and human health. PLoS One. 2017; 12(7): e0180599. Published online 2017 Jul 3. doi: 10.1371/journal.pone.0180599

[53] Matthew A. Croxen, Robyn J. Law, Roland Scholz, Kristie M. Keeney, Marta Wlodarska, B. Brett Finlay. Recent Advances in Understanding Enteric Pathogenic Escherichia coli. Clin Microbiol Rev. 2013 Oct; 26(4): 822-880. doi: 10.1128/CMR.00022-13

[54].Kaper JB, Nataro JP, Mobley HL. 2004. Pathogenic Escherichia coli. Nat. Rev. Microbiol.2:123-140

[55].Croxen MA, Finlay BB. 2010.Molecular mechanisms of Escherichia coli pathogenicity. Nat. Rev. Microbiol.8:26-38

[56]. Edyta Golińska, Anna Tomusiak, Tomasz Gosiewski, Grażyna Więcek, Agnieszka Machul, Diana Mikołajczyk, Małgorzata Bulanda, Piotr B Heczko, Magdalena Strus. Virulence factors of Enterococcus strains isolated from patients with inflammatory bowel disease. World J Gastroenterol.2013 Jun 21;19(23): 3562-3572.Published online 2013 Jun 21.doi:10.3748/wjg.v19.i23.3562

[57].Raffael C. Inglin, Leo Meile, Marc J. A. Stevens. Clustering of Pan- and Core-genome of Lactobacillus provides Novel Evolutionary Insights for Differentiation. BMC Genomics. 2018; 19: 284.Published online 2018 Apr 24.doi:10.1186/s12864-018-4601-5

[58]. eurath MF, Finotto S, Glimcher LH. The role of Th1/Th2 polarization in mucosal immunity. Nat Med. 2002;8(6):567-73. doi:10.1038/nm0602-567.

[59].Yohei Kanamori, Masaru Murakami§̧, Makoto Sugiyama, Osamu Hashimoto, Tohru Matsui, Masayuki Funabał.Interleukin-1ß (IL-

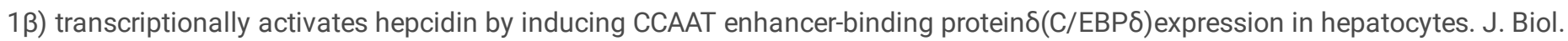
Chem. (2017) 292(24) 10275-10287

[60].P.T. Lee, S. Bird, J. Zou, S.A.M. Martin.Phylogeny and expression analysis of C-reactive protein (CRP) and serum amyloid-P (SAP) like genes reveal two distinct groups in fish. Fish Shellfish Immunol. 2017 Jun; 65: 42-51.doi:10.1016/j.fsi.2017.03.037

[61].Manu Sudhakar, Santhi Silambanan, Abhinand S. Chandran, Athira A. Prabhakaran, Ramya Ramakrishnan.C-Reactive Protein (CRP) and Leptin Receptor in Obesity: Binding of Monomeric CRP to Leptin Receptor.Front Immunol.2018;9: 1167.Published online 2018 May 29. doi: 10.3389/fimmu.2018.01167

[62].Chi-Dung Yang, Hsi-Yuan Huang, Sirjana Shrestha, et al. Large-Scale Functional Analysis of CRP-Mediated Feed-Forward Loops.Int J Mol Sci. 2018 Aug;19(8): 2335. Published online 2018 Aug 9.doi:10.3390/ijms19082335

[63].Ann Kathrin Heroven, Maike Sest, Fabio Pisano,et al.Front Cell Infect Microbiol.2012;2:158. Published online 2012 Dec 17.doi:10.3389/fcimb.2012.00158

\section{Tables}




\begin{tabular}{lllll}
\hline & Weight & Food intake & Water intake & Stool in $2 \mathrm{~h}$ \\
\hline A & $204.02 \pm 13.5$ & $19.02 \pm 3.63$ & $38.86 \pm 4.73$ & $0.96 \pm 0.48$ \\
B & $203.31 \pm 17.2$ & $19.58 \pm 2.83$ & $49.76 \pm 11.31$ & $2.20 \pm 1.38$ \\
C & $208.09 \pm 22.15$ & $18.93 \pm 2.75$ & $44.40 \pm 9.24$ & $2.23 \pm 1.09$ \\
D & $188.13 \pm 24.05$ & $15.47 \pm 5.66$ & $41.96 \pm 18.95$ & $2.47 \pm 1.53$ \\
E & $209.00 \pm 18.58$ & $18.80 \pm 2.11$ & $60.33 \pm 9.02$ & $2.82 \pm 2.01$ \\
F & $210.70 \pm 19.18$ & $20.31 \pm 3.57$ & $52.68 \pm 10.23$ & $3.09 \pm 1.79$ \\
G & $212.12 \pm 20.31$ & $20.66 \pm 3.13$ & $53.47 \pm 7.84$ & $2.33 \pm 1.55$ \\
\hline$F$ & 2.313 & 2.807 & 6.213 & 3.043 \\
$P$ & 0.04 & 0.016 & $<0.001$ & 0.009 \\
\hline
\end{tabular}

Table2 Comparison of total nine microbiota for A-G groups on involved days (Bacteria Number *E6)

\begin{tabular}{rrrrrr}
\hline & N & Mean & SD & \multicolumn{2}{c}{$95 \%$ CI } \\
& & & & Lower & Upper \\
\hline A & 432 & 1376.7 & 3683.8 & 562.2 & 2191.3 \\
B & 432 & 687.1 & 1498.7 & 355.7 & 1018.5 \\
C & 432 & 1474.9 & 4187.5 & 549.0 & 2400.8 \\
D & 432 & 478.2 & 1758.1 & 65.0 & 891.3 \\
E & 432 & 664.5 & 1567.9 & 317.8 & 1011.2 \\
F & 432 & 403.8 & 1172.0 & 144.6 & 662.9 \\
G & 432 & 3609.8 & 21206.5 & -1079.3 & 8298.9 \\
\hline F & & & & & 1.432 \\
P & & & & & $<.001 * *$ \\
\hline
\end{tabular}

Table 3 Comparison of every microbiota after weighted analysis for all days from A to G (Mean,*E6)

Microbiota by weighted analysis and croostabs

\begin{tabular}{|c|c|c|c|c|c|c|c|c|c|c|c|}
\hline & & \multicolumn{9}{|c|}{ Microbiota by weighted analysis and croostabs } & \multirow[t]{2}{*}{$\mathrm{P}$} \\
\hline & & Staphylococcus aureus & Bifidobacterium & Yeast & Bacteroides & Clostridium & Anaerobic bacteria & E.coli & Enterococcus & Lactobacillus & \\
\hline \multirow[t]{7}{*}{ group } & A & 1244 & 60400 & 0 & 3822 & 860 & 8814 & 1954 & 354 & 31510 & \multirow[t]{7}{*}{$<0.001 *$} \\
\hline & B & 6625 & 22786 & 0 & 2578 & 169 & 6927 & 2715 & 6678 & 5916 & \\
\hline & $\mathrm{C}$ & 5028 & 62940 & 0 & 1345 & 603 & 5250 & 3907 & 7116 & 17162 & \\
\hline & $\mathrm{D}$ & 5740 & 22581 & 0 & 575 & 156 & 2981 & 2556 & 2710 & 2505 & \\
\hline & $\mathrm{E}$ & 5454 & 21400 & 36 & 379 & 1456 & 2860 & 8518 & 2412 & 7900 & \\
\hline & $\mathrm{F}$ & 4476 & 12106 & 1 & 270 & 3786 & 3564 & 900 & 3467 & 4320 & \\
\hline & G & 4303 & 78950 & 0 & 225 & 6004 & 3732 & 7669 & 4507 & 187000 & \\
\hline
\end{tabular}

Table 4 Comparison of every microbiota in different day from A to G ( Mean , *E6) 


\begin{tabular}{|c|c|c|c|c|c|c|c|c|c|c|c|}
\hline \multicolumn{2}{|c|}{ Group and days } & \multicolumn{9}{|c|}{ Microbiota for weighted analysis and crosstabs } & \multirow[t]{2}{*}{ P-Value } \\
\hline & & Staphylococcus aureus & Bifidobacterium & Yeast & Bacteroides & Clostridium & Anaerobic bacteria & E.coli & Enterococcus & Lactobacillus & \\
\hline $1^{\text {st }}$ & A & 170 & 36400 & 0 & 100 & 202 & 4150 & 20 & 16 & 15000 & $<0.001$ \\
\hline \multirow[t]{6}{*}{ day } & $\mathrm{B}$ & 1302 & 6100 & 0 & 70 & 5 & 143 & 18 & 1 & 5200 & \\
\hline & $\mathrm{C}$ & 138 & 37200 & 0 & 11 & 80 & 2350 & 2 & 1 & 13340 & \\
\hline & $\mathrm{D}$ & 231 & 16900 & 0 & 4 & 1 & 2700 & 192 & 9 & 2503 & \\
\hline & $\mathrm{E}$ & 14 & 11500 & 0 & 2 & 1290 & 1400 & 3 & 2 & 6400 & \\
\hline & $\mathrm{F}$ & 76 & 9800 & 0 & 70 & 186 & 1164 & 15 & 7 & 2920 & \\
\hline & G & 3 & 67850 & 0 & 5 & 4 & 32 & 9 & 7 & 181700 & \\
\hline \multirow{7}{*}{$\begin{array}{l}4^{\text {th }} \\
\text { day }\end{array}$} & A & 3 & 4400 & 0 & 72 & 48 & 14 & 14 & 3 & 5000 & $<0.001$ \\
\hline & B & 1 & 566 & 0 & 14 & 1 & 0 & 266 & 0 & 0 & \\
\hline & $\mathrm{C}$ & 10 & 500 & 0 & 3 & 1 & 1 & 242 & 0 & 22 & \\
\hline & $\mathrm{D}$ & 0 & 206 & 0 & 0 & 0 & 0 & 184 & 0 & 0 & \\
\hline & $\mathrm{E}$ & 0 & 0 & 0 & 0 & 0 & 0 & 0 & 0 & 0 & \\
\hline & $\mathrm{F}$ & 0 & 0 & 0 & 0 & 0 & 0 & 0 & 0 & 0 & \\
\hline & G & 0 & 0 & 0 & 0 & 0 & 0 & 0 & 0 & 0 & \\
\hline \multirow{7}{*}{$\begin{array}{l}8^{\text {th }} \\
\text { day }\end{array}$} & $\mathrm{A}$ & 830 & 3700 & 0 & 2850 & 120 & 2600 & 1800 & 265 & 1940 & $\mathrm{P}<0.001$ \\
\hline & B & 2 & 1090 & 0 & 203 & 15 & 4 & 411 & 6 & 0 & \\
\hline & $\mathrm{C}$ & 0 & 1340 & 0 & 885 & 6 & 0 & 403 & 0 & 0 & \\
\hline & $\mathrm{D}$ & 0 & 675 & 0 & 0 & 10 & 0 & 610 & 0 & 0 & \\
\hline & $\mathrm{E}$ & 0 & 0 & 35 & 0 & 0 & 0 & 35 & 0 & 0 & \\
\hline & $\mathrm{F}$ & 0 & 0 & 0 & 0 & 0 & 0 & 0 & 0 & 0 & \\
\hline & G & 0 & 0 & 0 & 0 & 0 & 0 & 0 & 0 & 0 & \\
\hline \multirow{7}{*}{$\begin{array}{l}11^{\text {th }} \\
\text { day }\end{array}$} & $\mathrm{A}$ & 161 & 8400 & 0 & 650 & 200 & 150 & 51 & 21 & 1870 & $\mathrm{P}<0.001$ \\
\hline & B & 1620 & 8130 & 0 & 920 & 380 & 2080 & 720 & 1670 & 395 & \\
\hline & $\mathrm{C}$ & 780 & 14800 & 0 & 2940 & 570 & 899 & 1360 & 1214 & 700 & \\
\hline & $\mathrm{D}$ & 342 & 4800 & 0 & 3 & 646 & 281 & 1570 & 1621 & 20 & \\
\hline & $\mathrm{E}$ & 1740 & 7600 & 0 & 0 & 730 & 860 & 480 & 1010 & 0 & \\
\hline & $\mathrm{F}$ & 1520 & 6000 & 0 & 0 & 970 & 960 & 555 & 2200 & 0 & \\
\hline & G & 1600 & 18000 & 0 & 0 & 1500 & 1300 & 2960 & 2500 & 1400 & $\mathrm{P}<0.001$ \\
\hline \multirow{7}{*}{$\begin{array}{l}14^{\text {th }} \\
\text { day }\end{array}$} & A & 80 & 7500 & 0 & 150 & 150 & 1900 & 69 & 4900 & 7700 & \\
\hline & B & 3700 & 6900 & 0 & 800 & 110 & 4700 & 1300 & 5000 & 320 & \\
\hline & $\mathrm{C}$ & 4100 & 9100 & 0 & 600 & 500 & 15000 & 1900 & 5900 & 3100 & \\
\hline & $\mathrm{D}$ & 3740 & 6542 & 0 & 500 & 720 & 3450 & 2570 & 6451 & 4432 & \\
\hline & $\mathrm{E}$ & 3700 & 2300 & 1 & 760 & 3260 & 3600 & 8000 & 1400 & 1500 & \\
\hline & $\mathrm{F}$ & 4400 & 2300 & 1 & 3600 & 3600 & 2400 & 880 & 3400 & 1400 & \\
\hline & G & 2700 & 14100 & 0 & 2200 & 4500 & 2400 & 4700 & 3300 & 3900 & \\
\hline
\end{tabular}

Table 5 Comparison of inflammation factors from group A to group $\mathrm{G}(\mathrm{pg} / \mathrm{ml})$ 


\begin{tabular}{|c|c|c|c|c|c|c|c|c|}
\hline & & \multirow[t]{2}{*}{$\mathrm{N}$} & \multirow[t]{2}{*}{ Mean } & \multirow[t]{2}{*}{ SD } & \multicolumn{2}{|c|}{ 95\% Confidence Interval for Mean } & \multirow[t]{2}{*}{$\mathrm{F}$} & \multirow[t]{2}{*}{$\mathrm{P}$} \\
\hline & & & & & Lower Bound & Upper Bound & & \\
\hline \multirow[t]{7}{*}{ TNF } & A & 12 & 179.1 & 25.9 & 162.7 & 195.8 & 204.89 & $<0.001^{* *}$ \\
\hline & B & 12 & 207.0 & 12.0 & 199.4 & 214.6 & & \\
\hline & $\mathrm{C}$ & 12 & 244.1 & 10.8 & 237.2 & 251.0 & & \\
\hline & $\mathrm{D}$ & 12 & 272.3 & 18.3 & 260.7 & 283.9 & & \\
\hline & $\mathrm{E}$ & 12 & 276.0 & 8.3 & 270.8 & 281.3 & & \\
\hline & $\mathrm{F}$ & 12 & 311.8 & 9.0 & 306.0 & 317.9 & & \\
\hline & G & 12 & 361.4 & 12.2 & 353.6 & 369.2 & & \\
\hline \multirow[t]{7}{*}{ IL1 $\beta$} & $\mathrm{A}$ & 12 & 65.8 & 19.2 & 53.5 & 78.0 & 341.57 & $<0.001^{* *}$ \\
\hline & B & 12 & 100.3 & 8.4 & 94.9 & 105.6 & & \\
\hline & $\mathrm{C}$ & 12 & 130.6 & 12.5 & 122.7 & 138.6 & & \\
\hline & $\mathrm{D}$ & 12 & 178.2 & 11.4 & 171.0 & 185.4 & & \\
\hline & $\mathrm{E}$ & 12 & 218.8 & 36.5 & 195.7 & 242.1 & & \\
\hline & $\mathrm{F}$ & 12 & 308.2 & 12.6 & 300.2 & 316.2 & & \\
\hline & G & 12 & 345.8 & 22.3 & 331.7 & 360.0 & & \\
\hline \multirow[t]{7}{*}{ IL6 } & A & 12 & 124.0 & 24.8 & 108.2 & 139.8 & 354.15 & $<0.001^{* *}$ \\
\hline & B & 12 & 172.2 & 19.0 & 160.1 & 184.3 & & \\
\hline & $\mathrm{C}$ & 12 & 222.0 & 27.9 & 204.2 & 239.7 & & \\
\hline & $\mathrm{D}$ & 12 & 276.0 & 16.8 & 265.3 & 286.6 & & \\
\hline & $\mathrm{E}$ & 12 & 308.7 & 7.3 & 304.0 & 313.3 & & \\
\hline & $\mathrm{F}$ & 12 & 352.0 & 11.2 & 344.8 & 359.1 & & \\
\hline & G & 12 & 414.2 & 15.5 & 404.4 & 424.1 & & \\
\hline \multirow[t]{7}{*}{ CRP } & A & 12 & 9.8 & 2.8 & 8.0 & 11.6 & 1153.5 & $<0.001^{* *}$ \\
\hline & B & 12 & 15.9 & 2.2 & 14.5 & 17.3 & & \\
\hline & $\mathrm{C}$ & 12 & 25.7 & 2.7 & 24.0 & 27.4 & & \\
\hline & $\mathrm{D}$ & 12 & 35.9 & 1.8 & 34.7 & 36.9 & & \\
\hline & $\mathrm{E}$ & 12 & 48.7 & 1.9 & 47.5 & 49.8 & & \\
\hline & $\mathrm{F}$ & 12 & 57.2 & 1.6 & 56.2 & 58.2 & & \\
\hline & G & 12 & 71.2 & 2.8 & 69.4 & 72.92 & & \\
\hline
\end{tabular}

${ }^{*} \mathrm{p}<0.05$ indicate significant difference, ${ }^{* *} \mathrm{P}<0.001$

\section{Figures}



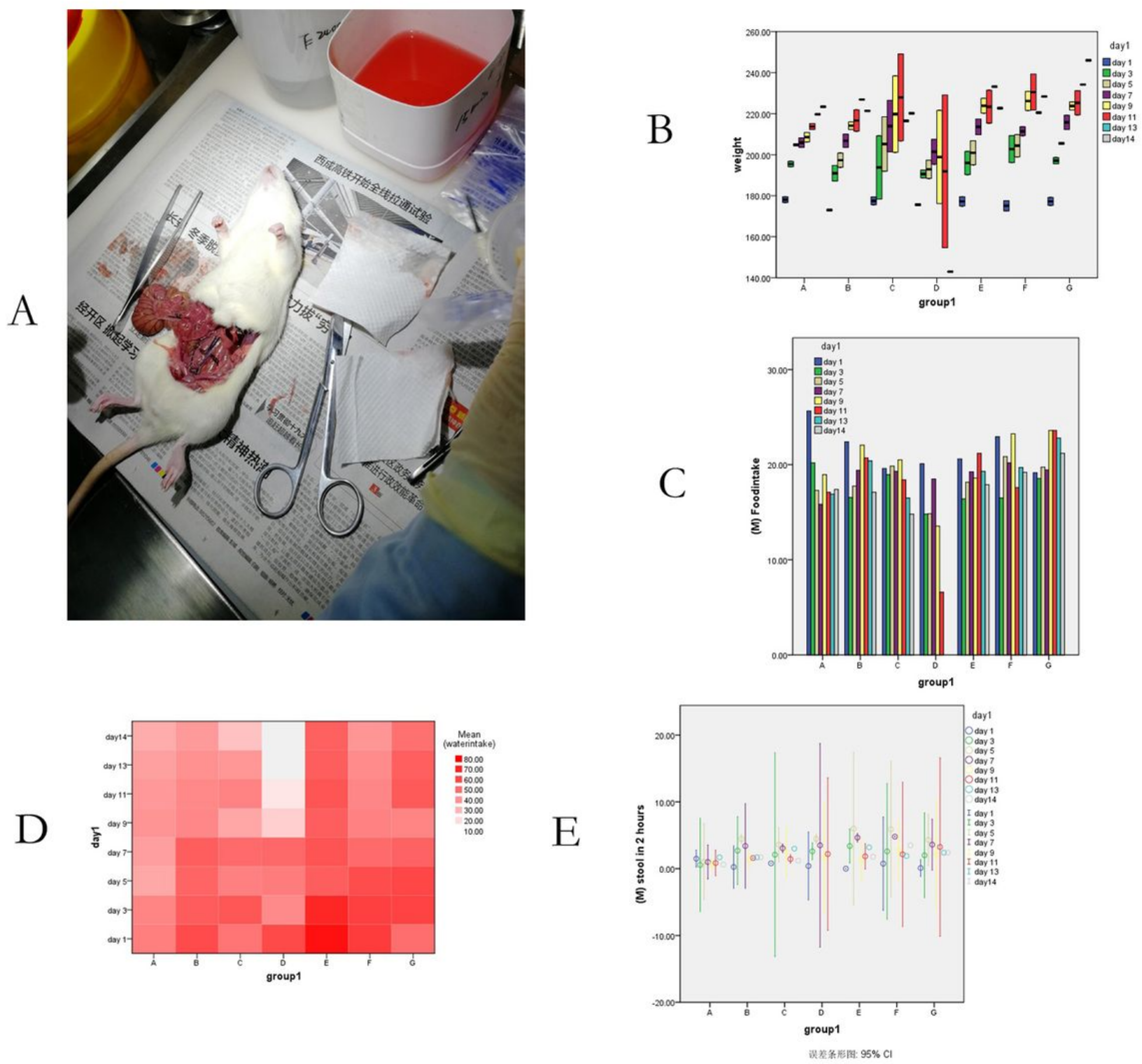

\section{Figure 1}

General picture of the rat. A: rat anatomical map; B: bar graph of different days of body weight in each group, $\mathrm{P}=0.04 ; \mathrm{C}$ : histogram of rats in each group on different days, $p=0.016$; $D$ : hot spot chart in different days of rats in each group comparing water consumption, $P<0.001$; E: scatter plots of stools in different groups of rats for 2 hours in different days, $P=0.009$. 


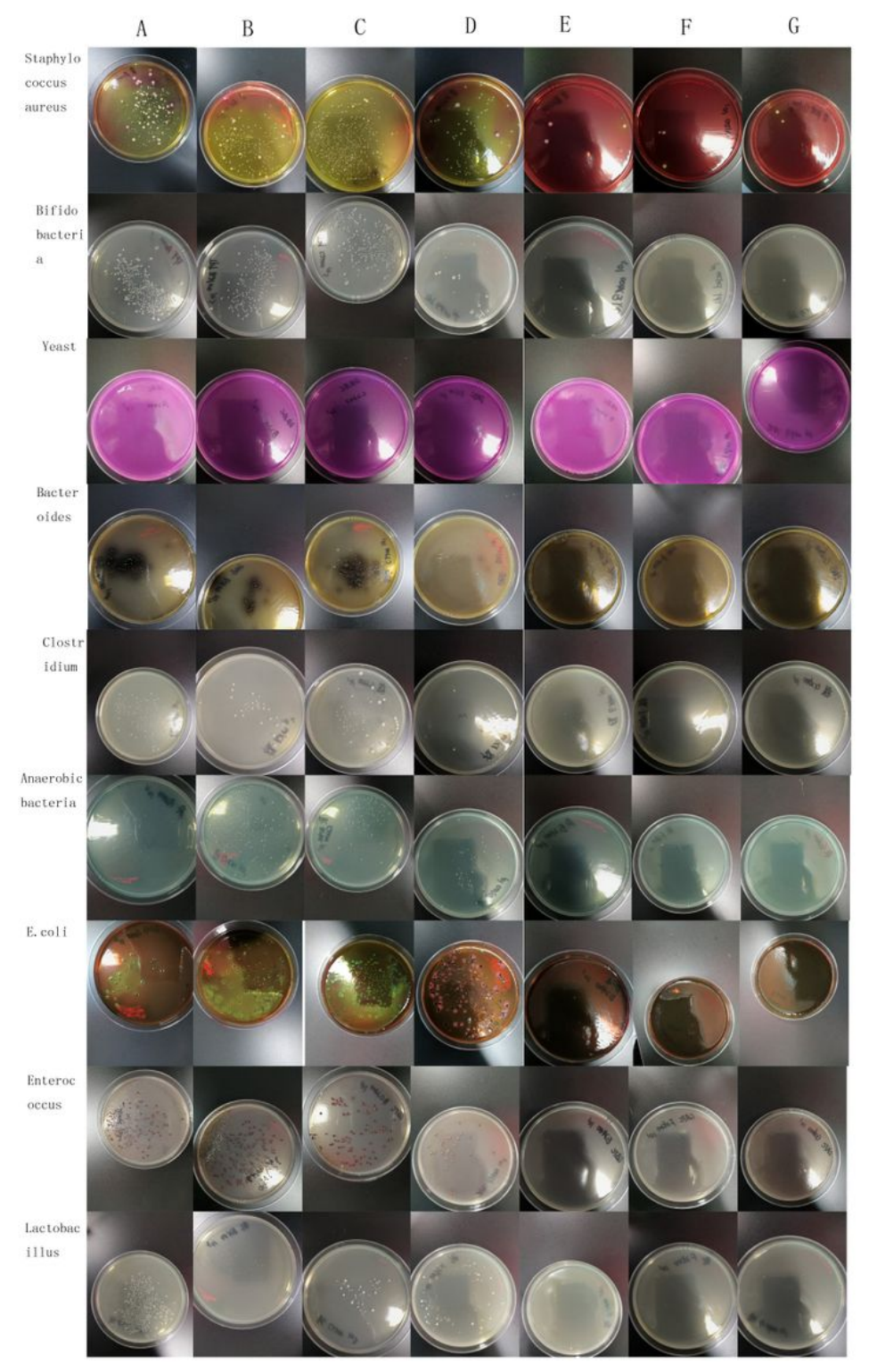

\section{Figure 2}

On the fourth day, 9 kinds of microbiota culture pictures. Mannitol sodium chloride agar medium (culture of Staphylococcus aureus); TPY agar medium (culture of Bifidobacterium); DRBC agar (cultured yeast); Bacteroides-bile-escin agar (BBE) (culture of Bacteroides); reinforced cloaca culture medium (culture of Clostridium ); anaerobic agar (culture of anaerobic bacteria); Eosin blue agar medium (EMB) (culture of E. coli); CATC agar (cultured Enterococcus); lactic acid Bacillus selective agar (cultured Lactobacillus) 
A $\quad$ B $\quad$ C
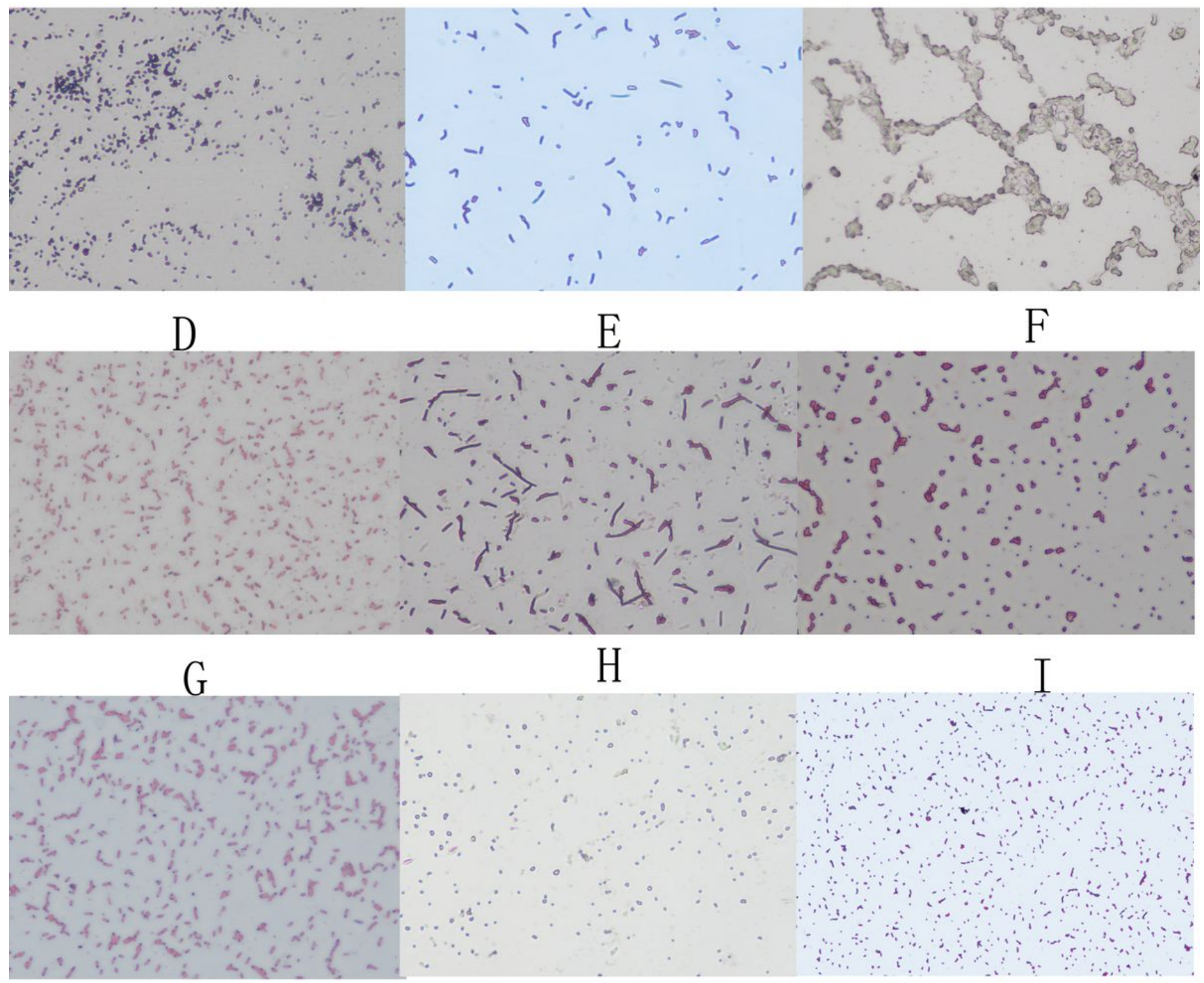

\section{Figure 3}

A picture of counted microbiota specimen under a microscope on fourth day. A:Staphylococcus aureus; B:Bifidobacterium;C:yeast;

D:Bacteroides E:Clostridium; F:anaerobic bacteria; G: E. Coli; H:Enterococcus; I: Lactobacillus 


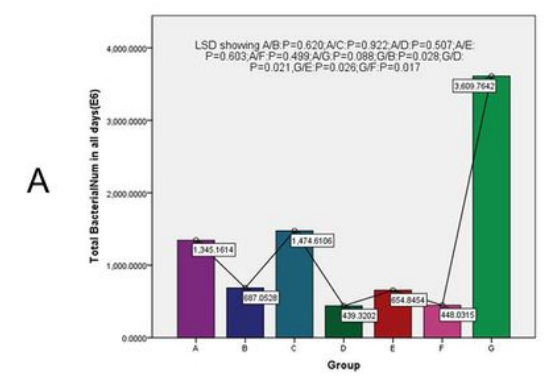

B
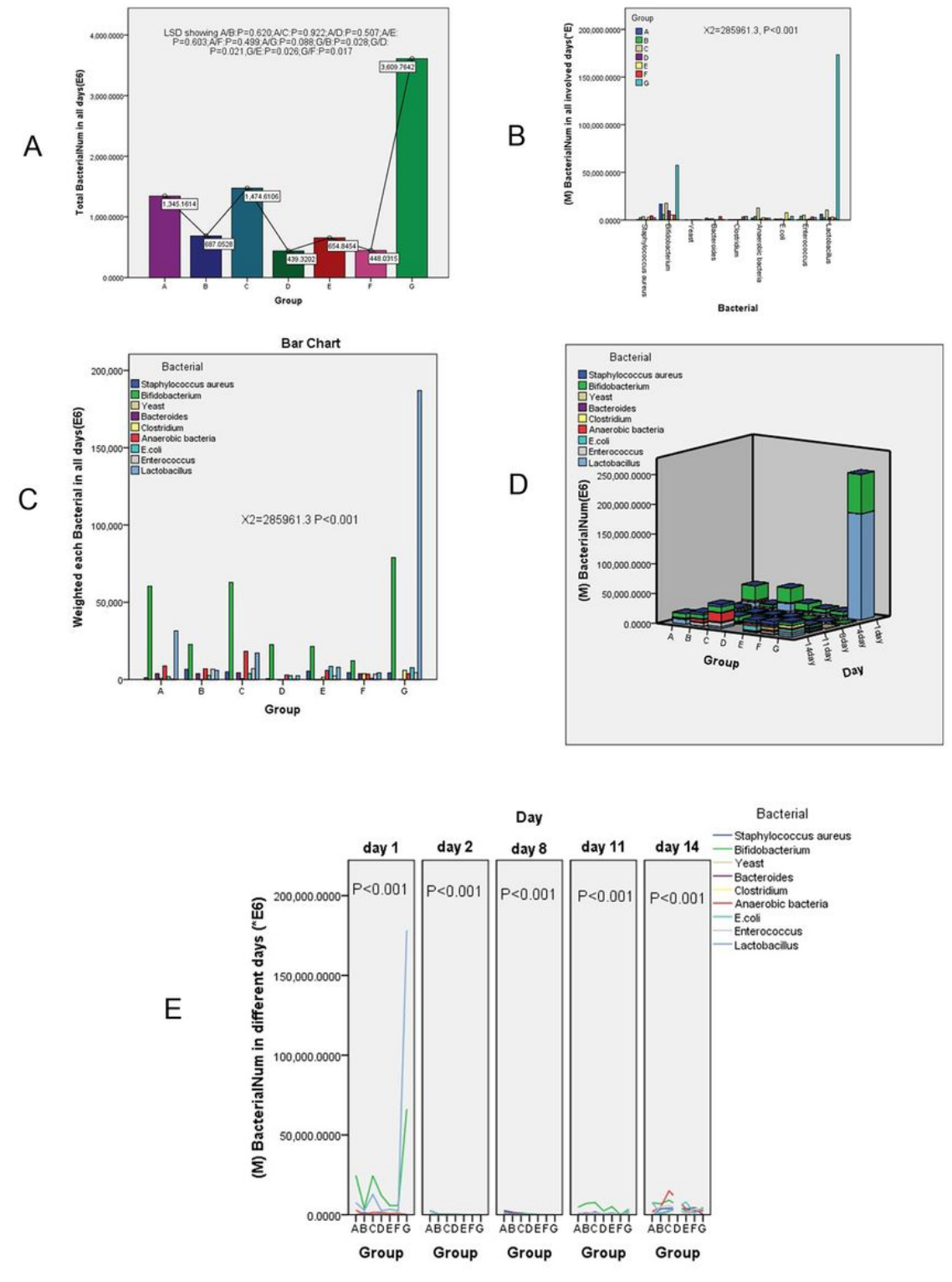

\section{Figure 4}

9 kinds of microbiota of all involved days were analyzed in different groups. A: Total microbiota in each group were compared using ANOVA and LSD, showing

A/B:P=0.620;A/C:P=0.922;A/D:P=0.507;A/E:P=0.603;A/F:P=0.499;A/G:P=0.088;G/B:P=0.028;G/D:P=0.021,G/E:P=0.026;G/F:P=0.017.B: different groups were compared in 9 microbiota, $P<0.001$; : 9 microbiota were weighted in different groups, $P<0.001 ; D$ : 3D maps of 9 microbiota in different groups and days, Bifidobacterium and Lactobacillus were significantly higher in the $G$ group on the first day than the other groups. E: Comparison of 9 microbiota between $A$ group to $\mathrm{G}$ group at first,4th,8th,11th, 14th days, all $\mathrm{P}<0.001$. 


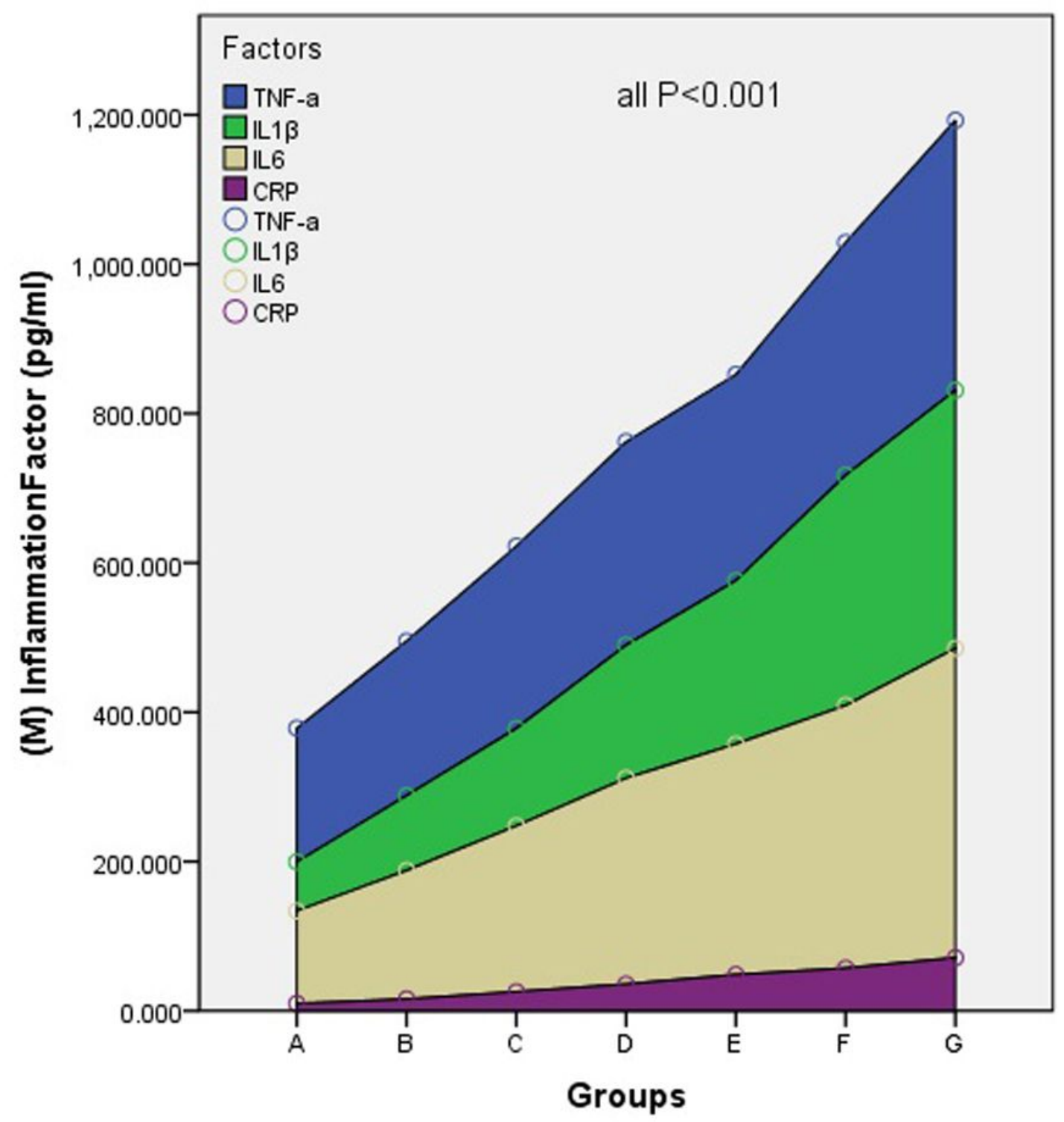

Figure 5

A regional map $(\mathrm{pg} / \mathrm{ml})$ of four inflammatory factors compared between groups. There were a significant difference about TNF$a, I L 1 \beta, I L-6$ and CRP between and within groups (all $P<0.001$ ). And the mean values from high to low are TNF- $a, I L 1 \beta, I L-6, C R P$.

\section{Supplementary Files}

This is a list of supplementary files associated with this preprint. Click to download.

- NC3RsARRIVEGuidelines1.pdf 